$16^{\text {th }}$ International Conference on

AEROSPACE SCIENCES \& AVIATION TECHNOLOGY,

ASAT - 16 - May 26 - 28, 2015, E-Mail: asat@ mtc.edu.eg

Military Technical College, Kobry Elkobbah, Cairo, Egypt

Tel : +(202) 24025292 - 24036138, Fax: +(202) 22621908

\title{
Characterizing the Degrading Effects of Scattering by Atmospheric Fog on the Spatial Resolution of Imaging Systems
}

\author{
Mohamed Elsayed Abdelhady Hanafy, PhD Fawzy Eltohamy Hassan, PhD \\ Egyptian Armed Forces
}

\begin{abstract}
In this paper the effects of scattering, with four atmospheric fog aerosols models, on the overall point spread function (PSF) of an imaging system are investigated. The PSF and radiometric calculations are then used to estimate the mean numbers of direct path and scattered photons detected by the imaging system in the visible and near infra-red (NIR) spectral bands of the electro-magnetic spectrum (EMS), for a horizontal near-ground imaging scenario. The image spectrum signal-to-noise ratio (SNR) is then used to estimate the noise effective spatial resolution of the imaging system which emphasizes the degrading effect of fog aerosol models on the spatial resolution of the imaging systems.
\end{abstract}

Key words: scattering, atmospheric fog aerosols, point spread function, signal-to-noise ratio, spatial resolution.

\section{Introduction}

The propagation of electro-magnetic radiation (EMR) within the Earth's atmosphere depends on the wavelength of the radiation, $\lambda$, and the nature of the medium being traversed [1]. This medium contains various molecular species and aerosol particles whose composition and density are the determining factors in the amount of radiation detected by an electro-optical imaging system. EM fields propagating through the atmosphere are either attenuated or blurred. Attenuation is attributed to absorption and large angle scattering (Rayleigh scattering) caused by atmospheric molecules of small size compared to the propagated wavelength, $r<<$ $\lambda$, where $r$ is the particulate radius. Blurring is attributed to optical turbulence and small-angle forward scattering (Mie scattering) caused by large sized aerosols, $r \geq \lambda$, which is known as the adjacency effect, since photons are imaged in pixels adjacent to those in which they ought to have been imaged [2]. Scattering has the effect of de-correlating the light leaving the target from the unscattered light reaching the imaging system; i.e., the scattered and direct path radiation received at the imaging system entrance pupil, after propagation through a scattering medium, are uncorrelated [푼. $\underline{]}$. 
Also, scattering has the effect of broadening the angle at which the scattered light arrives at the imaging system compared to the unscattered light . In this paper, four atmospheric fog aerosols, which are characterized by strong small-angle forward scattering, will be modeled in the second section using Mie scattering theory; where the physical parameters of these aerosol models, including their particle compositions and size distribution function, are utilized to calculate the optical properties of the various fog models. The average PSF, which is the basic physical function that describes the spatial response of an imaging system, due to scattering by various fog aerosol models will be computed in the third section. This PSF properly accounts for the effects of the diffraction, scattering, and the appropriate optical power level of both the unscattered and the scattered radiation arriving at the pupil of the imaging system. In the fourth section we calculate the image spectrum SNR in the visible, and NIR bands. The calculation is based on a radiometric technique for estimating the average number of photons detected by the imaging system, for a horizontal near-ground imaging scenario, in combination with the calculated PSFs. Conclusions are presented in the fifth section. Our key result is the demonstration of a technique for predicting the resolution limit of an imaging system working in the presence of fog aerosols as a function of the optical system parameters, the scattering medium, and the signal level.

\section{Atmospheric Fog Aerosol Models}

Aerosol modeling has two goals: the first is the accurate representation of the aerosol distribution (density) by the size distribution function, and representing the aerosol composition by the spectral refractive index. The second goal is the accurate calculation of the optical properties of the whole medium including the atmospheric optical depth, EM attenuation coefficients, and total albedo of the medium $[\underline{5}, \underline{6}]$. The investigated fog models include: heavy advection, moderate advection, heavy radiation, and moderate radiation fog. In this section, the physical parameters of these aerosol models, including their particle compositions and size distribution function, are utilized to calculate their optical properties.

\subsection{Particle size distribution function}

Aerosol particle size distribution function, $n(r)$, used to model fog aerosols is the modified Gamma distribution function $[\underline{7}, \underline{8}]$ which is given by:

$$
n(r)=A r^{\alpha} \exp \left(-B r^{\gamma}\right)
$$

where $A, B, \alpha$ and $\gamma$ are fit coefficients of the distribution. Table (1) lists the characteristic parameters corresponding to various relative humidity $(\mathrm{RH})$, and meteorological range $(M)$ values $[m]$, of the fog aerosol models. The meteorological range corresponds to the range between the target and the imaging system where the contrast of the detected target is reduced to $2 \%$ [9]. Figure (1) shows the size distribution functions of the four atmospheric fog aerosol models in accordance with the data listed in Table (1). 
Table (1) Fog aerosol models characteristic parameters: Modified Gamma size distribution function $[\underline{6}, \underline{7}]$.

\begin{tabular}{lccccc}
\hline \multirow{2}{*}{ Fog aerosol models } & \multicolumn{5}{c}{ Size distribution parameters } \\
\cline { 2 - 6 } & $M[m]$ & $A$ & $B$ & $\alpha$ & $\gamma$ \\
\hline Heavy Advection & 136 & 0.027 & 0.3 & 3 & 1 \\
Moderate Advection & 211 & 0.06592 & 0.375 & 3 & 1 \\
Heavy Radiation & 235 & 2.37305 & 1.5 & 6 & 1 \\
Moderate Radiation & 453 & 607.5 & 3 & 6 & 1 \\
\hline
\end{tabular}

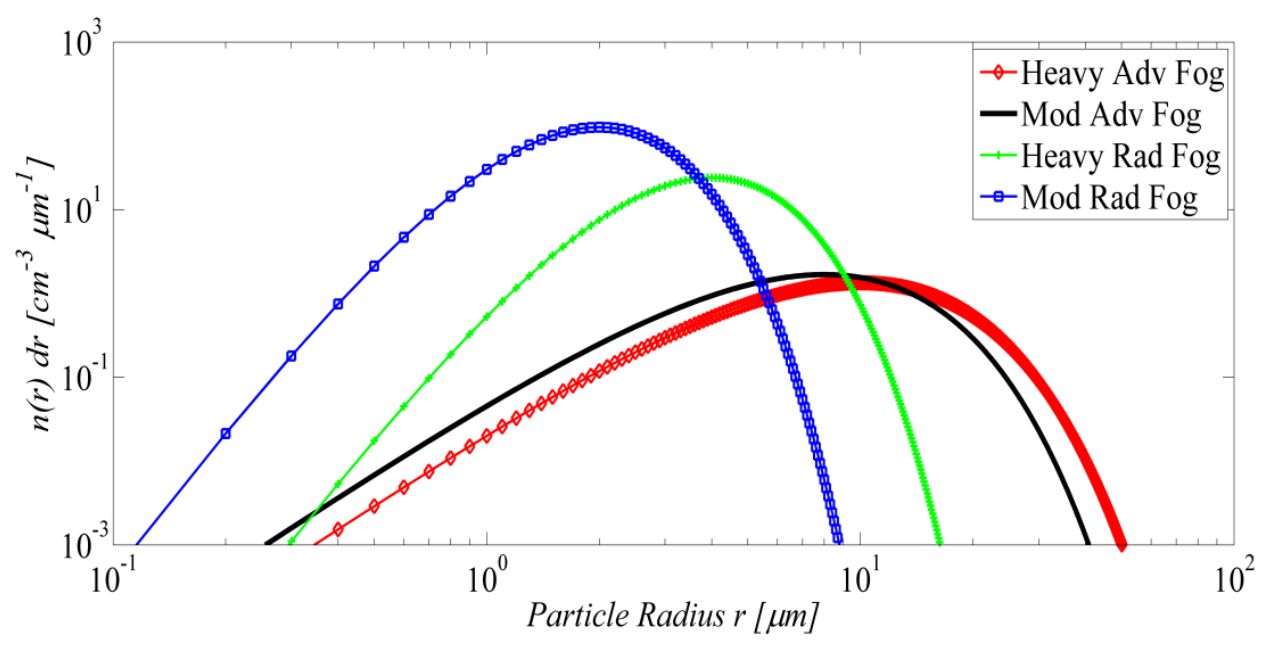

Figure (1) Modified Gamma size distribution function for fog aerosol models in accordance with the data listed in Table (1).

The data on the size distribution function and the spectral refractive indices for atmospheric aerosols, extracted from reference [7] in the visible and NIR bands, $\lambda=0.39-2[\mu \mathrm{m}]$, are used to calculate the optical properties of the atmospheric fog aerosol models.

\subsection{Atmospheric optical properties}

The optical properties of an atmospheric fog aerosol model which are used to characterize the PSF of an imaging system include the atmospheric optical depth (AOD), $\tau$, and the total albedo, $\omega_{o}^{t}(\lambda)$, which indicates the probability that an intercepted photon will be scattered rather than being absorbed. The AOD expresses the power density removed from a propagating wave through the atmosphere upon scattering or absorption [10],

$$
\tau(\lambda)=\int k_{e x t}(\lambda, z) d z
$$

where $z$ is the atmospheric path length $[m], k_{\text {ext }}$ is the atmospheric extinction coefficient $\left[\mathrm{m}^{-1}\right]$, which represents the relevant cumulative extinction cross sections of all particles in a unit volume of air [10], 


$$
k_{e x t}(\lambda)=k_{s c a}(\lambda)+k_{a b s}(\lambda)
$$

where $k_{s c a}$ and $k_{a b s}$ are the scattering and absorption coefficients $\left[m^{-1}\right]$, respectively. $k_{\text {ext }}(\lambda)$, $k_{s c a}(\lambda)$, and $k_{a b s}(\lambda)$ are given by [11],

$$
\begin{aligned}
& k_{e x t}(\lambda)=\int_{0}^{\infty} \sigma_{e x t}(\lambda, r) n(r) d r \\
& k_{s c a}(\lambda)=\int_{0}^{\infty} \sigma_{s c a}(\lambda, r) n(r) d r \\
& k_{a b s}(\lambda)=\int_{0}^{\infty} \sigma_{a b s}(\lambda, r) n(r) d r
\end{aligned}
$$

And the total albedo $\omega_{o}^{t}(\lambda)$ is given as the ratio of $k_{s c a}$ to $k_{\text {ext }}[\underline{10}, \underline{11]}$,

$$
\omega_{o}^{t}(\lambda)=\frac{k_{s c a}(\lambda)}{k_{e x t}(\lambda)}
$$

Plots of $k_{\text {ext }}(\lambda), k_{a b s}(\lambda)$, and $k_{s c a}(\lambda)$ for the fog aerosol models, in the visible and NIR bands $\lambda=$ $0.39-2[\mu \mathrm{m}]$, are shown in figure (2). The optical parameters of atmospheric fog aerosol models for various meteorological range $(M)$ values are calculated using our stand-alone Matlab code, which is based on Mie scattering theory, and are listed in Table (2). These optical properties are required to estimate the atmospheric transmittance and angular light scattering distribution, which contribute to the PSF of an imaging system as will be clarified in the next section.

\section{Point spread function (PSF) calculation}

The reduction in image quality is attributed either to reduction of image contrast, or to image blurring. The reduction of image contrast is due to atmospheric aerosols which cause extinction of the propagating radiation and scattering at large enough angles leading to an ultimate reduction in the SNR. While, the image blurring is due to small-angle scattering by atmospheric aerosols, where the scattered radiation collected by the sensor will be slightly deviated such that fine image features such as lines and edges are blurred. This spatial effect can be characterized as a broadening of the PSF [12]. Since the PSF is the basic physical function that describes the spatial response of an imaging system, its calculation accounts for the effects of the diffraction, scattering, and the appropriate optical power level of both the unscattered (direct) and the scattered radiation arriving at the pupil of the imaging system. Since the scattered radiation is temporally and spatially de-correlated from the direct radiation, we model the effects of the direct (coherent) radiation and the scattered (incoherent) radiation of the aerosol species as additive in the image plane. The angular distribution of the total detected PSF at the image plane, $\operatorname{PSF}_{t}(\beta)$, consists of the sum of two independent components $[\underline{10}, \underline{13}, \underline{14}]$,

$$
P S F_{\mathrm{t}}(\beta)=P S F_{d i r}(\beta)+P S F_{s c a}(\beta)
$$


where $\beta[\mathrm{rad}]$ is the angular distance in the image plane, $P S F_{d i r}(\beta)$ is the direct PSF, or the coherent intensity detected by the imaging system due to the direct radiation, and $P S F_{\text {sca }}(\beta)$ is the scattered PSF, or the incoherent intensity due to the scattered radiation. Considering a plane wave propagating in an aerosol-laden medium with path length $z$, and an imaging system having focal length $f$ and a circular entrance aperture of diameter $D$. In the case of an atmospheric model with an AOD greater than $1 ; \tau>1, P S F_{d i r}(\beta)$ may be written as [10],

$$
P S F_{d i r}(\beta)=e^{-\tau}\left(\frac{D}{2 f \beta}\right)^{2} J_{1}^{2}\left(\frac{k D \beta}{2}\right)
$$

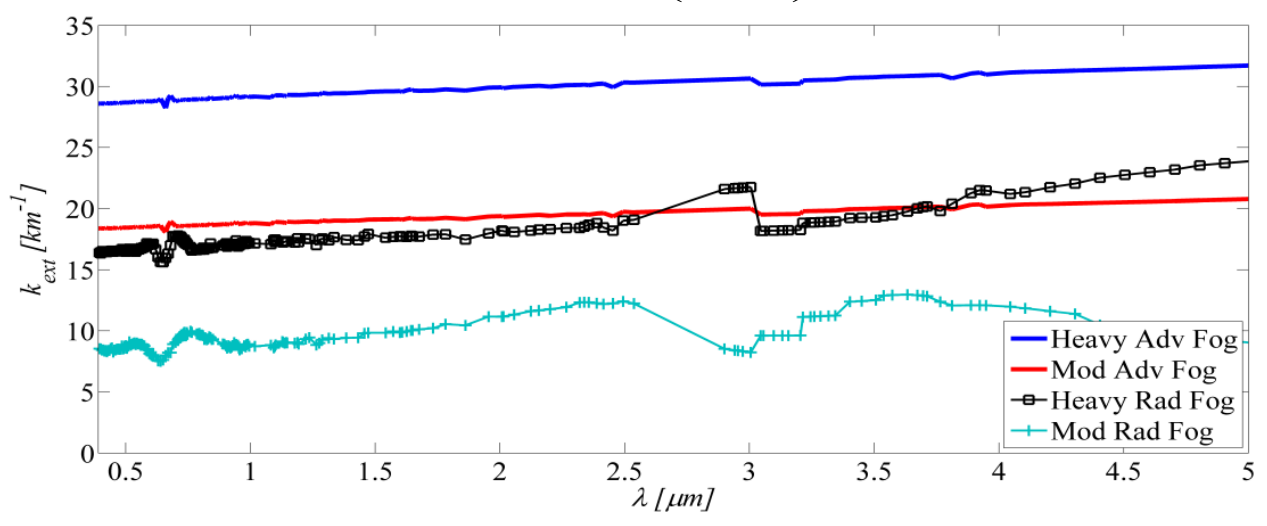

(a) Extinction coefficient $k_{\text {ext }}\left[\mathrm{km}^{-1}\right]$

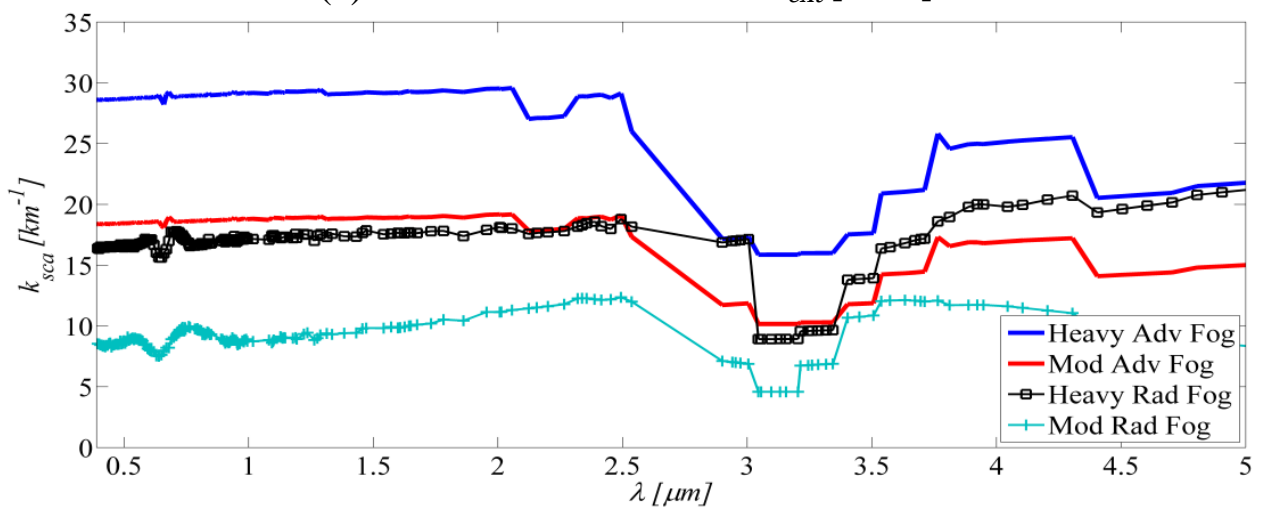

(b) Scattering coefficient $k_{s c a}\left[\mathrm{~km}^{-1}\right]$

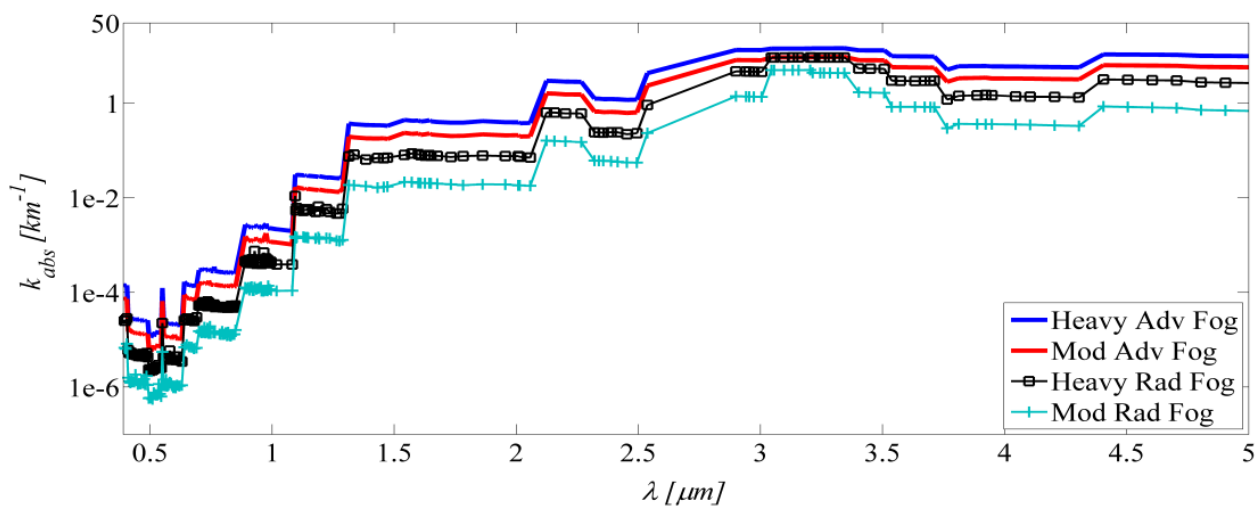

(c) Absorption coefficient $k_{a b s}\left[\mathrm{~km}^{-1}\right]$

Figure (2) Optical properties of atmospheric fog aerosol models. 
Table (2) Calculated optical properties of atmospheric fog aerosol models for various meteorological range $(M)$ values.

\begin{tabular}{lcccccc}
\hline \multirow{2}{*}{ Fog aerosol models } & $M[\mathrm{~m}]$ & \multicolumn{5}{c}{ Calculated optical properties } \\
\cline { 3 - 7 } & & $K_{a b s}\left[\mathrm{~km}^{-1}\right]$ & $K_{\text {sca }}\left[\mathrm{km}^{-1}\right]$ & $K_{\text {ext }}\left[\mathrm{km}^{-1}\right]$ & $\tau$ & $\omega_{o}^{t}(\lambda)$ \\
\hline Heavy Advection & 136 & $5.5 \times 10^{-5}$ & 28.413 & 28.7 & 14.37 & 0.99 \\
Moderate Advection & 212 & $3.8 \times 10^{-5}$ & 18.424 & 18.8 & 9.38 & 0.98 \\
Heavy Radiation & 235 & $7.8 \times 10^{-6}$ & 16.032 & 16.7 & 8.33 & 0.96 \\
Moderate Radiation & 453 & $1.3 \times 10^{-6}$ & 8.084 & 8.6 & 4.32 & 0.94 \\
\hline
\end{tabular}

where $J_{l}$ is Bessel function of the first kind of order 1 , and $k=2 \pi / \lambda$ is the wave number $\left[m^{-1}\right]$. $P F S_{\text {dir }}(\beta)$ is an attenuated Airy disc pattern. $P S F_{\text {sca }}(\beta)$ may be written as [10],

$$
P S F_{s c a}(\beta)=\left(\frac{D}{2 \rho_{i}}\right)^{2} \exp \left[-\tau\left(1-\omega_{o}^{t}\right)-\left(\frac{f \beta}{\rho_{i}}\right)^{2}\right]
$$

where $\rho_{i}$ is the radial spread of the scattered PSF at the image plane $[m]$, and is given by [10],

$$
\rho_{i}=0.26\left(\frac{\rho_{\text {Airy }} D}{\rho_{o}}\right)
$$

where $\rho_{\text {Airy }}=1.22 \lambda f / D$ is the radius of an Airy disk $[m]$, or the spatial spread of $P S F_{\text {dir }}(\beta)$ at the image plane, $\rho_{o}$ is the correlation distance of the wave incident upon the entrance aperture $[\mathrm{m}][\underline{10}]$,

$$
\rho_{o}=\frac{1.04 a_{\text {mode }}}{\sqrt{k_{\text {sca }} z}}
$$

where $a_{\text {mode }}$ is the modal radius of the size distribution $[m]$. From Eqs. (8-12), a final form of the total detected PSF may be written as,

$$
\operatorname{PSF}_{t}(\beta)=\frac{D^{2}}{4}\left\{\left(\frac{e^{-\tau}}{f^{2} \beta^{2}}\right) J_{1}^{2}\left(\frac{k D \beta}{2}\right)+\left(\frac{1}{\rho_{i}^{2}}\right) \exp \left[-\tau\left(1-\omega_{o}^{t}\right)-\left(\frac{f \beta}{\rho_{i}}\right)^{2}\right]\right\}
$$

A normalized total PSF is given by,

$$
P S F_{n}(\beta)=\frac{P S F_{d i r}(\beta)+P S F_{s c a}(\beta)}{P S F_{d i r}(0)+P S F_{s c a}(0)}
$$

Figure (3) shows plots of the angular distribution of the detected PSFs at the image plane for the fog aerosols models in accordance with the calculated optical parameters listed in Table (2). Figure (3.a) shows the 


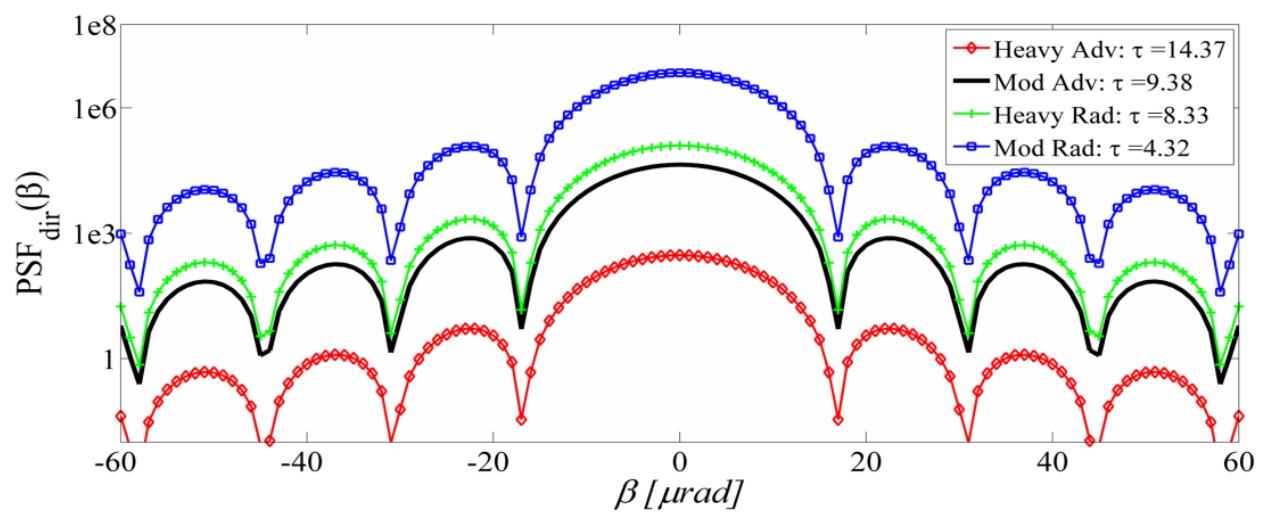

(a)

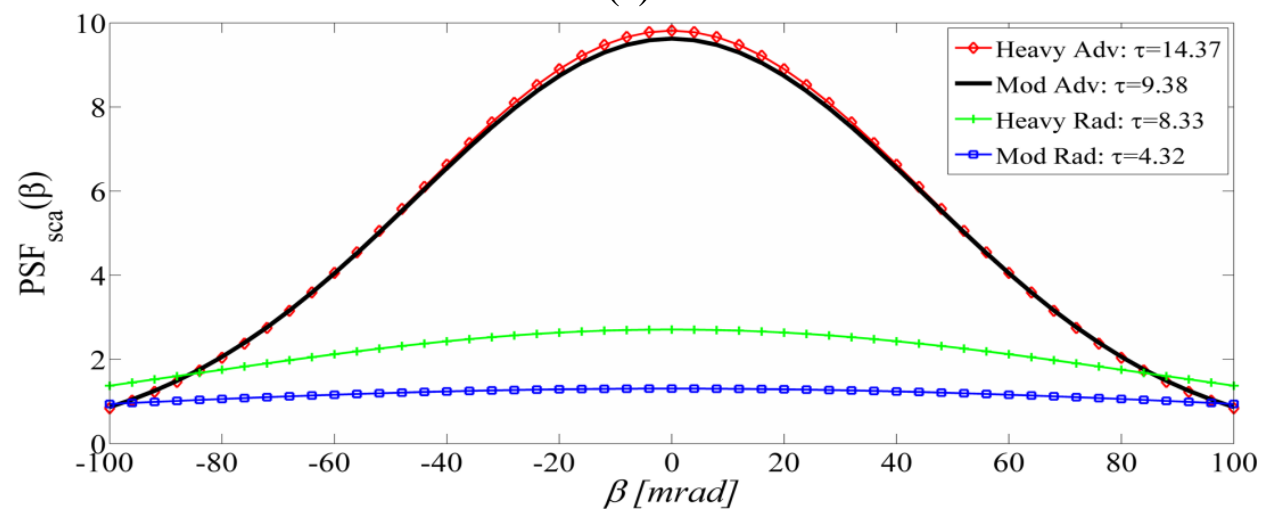

(b)

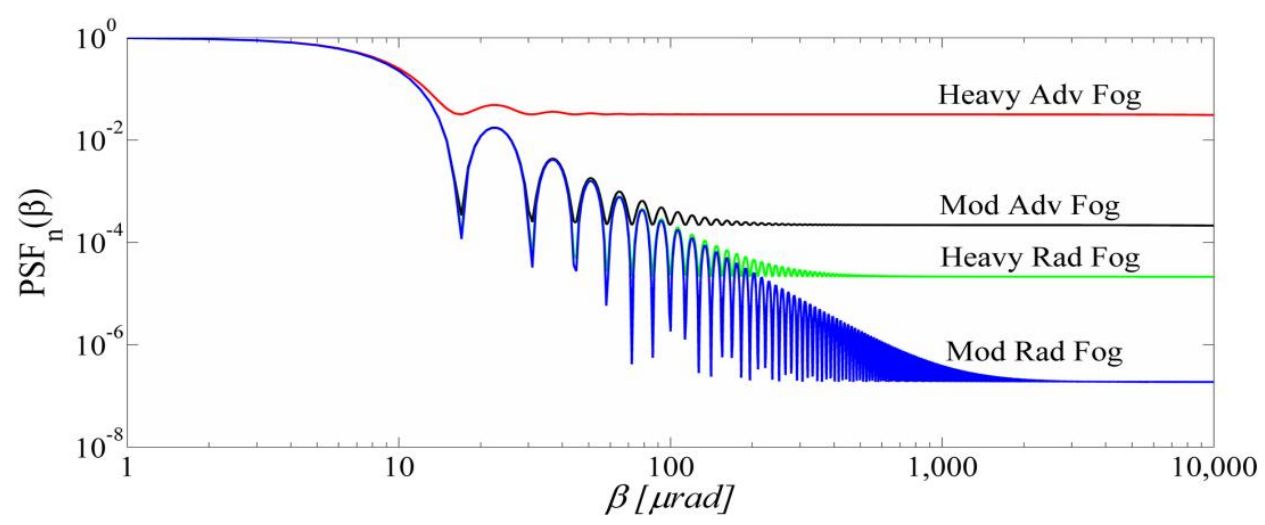

(c)

Figure (3) Angular distribution of the detected PSFs for atmospheric fog aerosol models in accordance with the calculated optical parameters listed in Table (2). Assuming an atmospheric path length $z=500[\mathrm{~m}]$, the imaging system focal length $f=10[\mathrm{~cm}]$, and the entrance aperture diameter $D=4[\mathrm{~cm}]$.

(a) Direct (coherent) PSF, (b) Scattered (incoherent) PSF, (c) Normalized total PSF. angular distribution of the direct PSF, $P S F_{d i r}(\beta)$, figure (3.b) shows the radial distribution of the scattered PSF, $P S F_{s c a}(\beta)$, and figure (3.c) shows the radial distribution of the normalized total PSF, $P S F_{n}(\beta)$. It is apparent that upon increased values of the AOD, $\tau$, due to the various fog aerosol models, the level of $P S F_{\text {dir }}(\beta)$ decreases significantly, whereas the level of $P S F_{s c a}(\beta)$ increases. Also, $P S F_{\text {dir }}(\beta)$ is narrowly concentrated in an Airy disc pattern, while $P S F_{s c a}(\beta)$ is widely spread throughout the entire image plane, due to the broad angle at which the scattered light arrives at the imaging system pupil, compared to the direct light. Hence, $P S F_{n}(\beta)$ is composed of two main components: a narrow central peak, representing the direct radiation from the source, and a weaker wide-spread wing, representing the scattered radiation by each of 
the fog models. An important conclusion is that in the central portion of the image plane, region near $\beta=0$, as long as $P S F_{d i r}>P S F_{s c a}$, it could be possible to get a reasonable image of a distant source. However, for an increasing $\tau$ values, as $P S F_{\text {sca }}(0)$ approaches $P S F_{d i r}(0)$ the image contrast decreases gradually until the image is completely unseen for $P S F_{s c a}(0) \geq P S F_{d i r}(0)$. A very important observation can be inferred from figure (3.c) is that the angular distance, $\beta$, at which the normalized total PSF becomes approximately flat, is the angle corresponding to the reciprocal of the cut-off frequency of an aerosol modulation transfer function (MTF). Considering the imaging system focal length of $10[\mathrm{~cm}]$ : For the heavy advection fog, moderate advection fog, heavy radiation fog, and moderate radiation fog, $\beta \approx 10,300,500,1000$ [urad], respectively, which is appropriate for an MTF cut-off frequencies of 100, 3.33, 2 and 1 [cycle/mrad]. Hence, for moderate radiation fog, the PSF is about 100 times broader than that of the heavy advection fog, and the MTF is about 100 times narrower. Aerosol MTF is thus for heavy advection fog a high spatial frequency phenomenon [3]. This confirms the effect of increased level of the aerosols scattered radiation on limiting the spatial resolution of an imaging system. However, the image spectrum SNR is another metric that will confirm the degrading effect of fog aerosols on limiting the spatial resolution of imaging systems as will be investigated in the next section.

\section{Image Spectrum Signal-to-Noise Ratio (SNR)}

let $I(v)$ be the Fourier transform of an image which has been detected with a photon counting camera, where $v$ is the spatial frequency [cycle/rad]. The image spectrum SNR is defined as the ratio of the mean of $I(v)$ to the standard deviation of $I(v)$ [15]. The image spectrum SNR is a measure of the relative strengths of the image spectrum signal and the random variability of the signal in the spatial frequency domain. Moreover, the image spectrum SNR provides a means of estimating the noise effective resolution of an imaging system, hence providing a useful metric of evaluating the effects of various sources of noise on the image quality. The SNR of an image obtained with a deterministic MTF and a photon-limited image detection system is given by [4],

$$
S N R(v)=\frac{K_{d i r}\left|H(v) O_{n}(v)\right|}{\sqrt{K_{d i r}+K_{s c a}}},
$$

where $\operatorname{SNR}(v)$ is the image spectrum SNR, $H(v)$ is the MTF of the imaging system, $O_{n}(v)$ is the normalized Fourier spectrum of the target irradiance distribution, $K_{d i r}(\lambda)$ is the mean number of photons directly transmitted to the imaging system from the target, and $K_{\text {sca }}(\lambda)$ is the mean number of photons scattered by the atmospheric aerosols before reaching the imaging system. $K_{d i r}(\lambda)$ and $K_{s c a}(\lambda)$ are given by [4]],

$$
K_{d i r}(\lambda)=\left[\frac{P S F_{d i r}(0, \lambda)}{P S F_{t}(0, \lambda)}\right] K_{\text {avg }}(\lambda),
$$


Where $\operatorname{PSF}_{d i r}(0, \lambda), P S F_{s c a}(0, \lambda)$ and $\operatorname{PSF}_{t}(0, \lambda)$ are the direct, scattered, and total effective PSF,

$$
K_{s c a}(\lambda)=\left[\frac{P S F_{s c a}(0, \lambda)}{P S F_{t}(0, \lambda)}\right] K_{a v g}(\lambda)
$$

respectively, at the central portion of the imaging plane; $\operatorname{PSF}_{t}(0, \lambda)=P S F_{d i r}(0, \lambda)+$ $P S F_{\text {sca }}(0, \lambda)$, and $K_{\text {avg }}(\lambda)$ is the average number of photons detected by the imaging system is given by [4],

$$
K_{\text {avg }}(\lambda)=\frac{A A_{\text {aper }} \tau(\lambda) \tau_{o p} \eta t_{\text {int }} \lambda M(\lambda)}{\pi h c z^{2}}
$$

Where $A$ is the area of the target $\left[\mathrm{m}^{2}\right], A_{\text {aper }}=\pi D^{2} / 4\left[\mathrm{~m}^{2}\right]$, is the area of the entrance aperture of the imaging system, $\tau_{o p}$ is the optics transmittance, $\eta$ is the quantum efficiency of the detectors, $t_{\text {int }}$ is the integration time of the detectors $[s], M(\lambda)$ is the radiant exitance of the target in the infinitesimal wavelength interval $(\lambda, \lambda+\Delta \lambda)\left[W^{-2}\right], h$ is Planck's constant, $h=6.626 \times 10^{-34}[J . s]$, and $c$ is the speed of light, $c=3 \times 10^{8}[\mathrm{~m} / \mathrm{s}]$. $K_{\text {avg }}$ may be calculated for the visible; $\lambda_{c}=$ $0.55[\mu \mathrm{m}]$ and NIR; $\lambda_{c}=1.22[\mu \mathrm{m}]$, spectral wavelength bands, where $\lambda_{c}$ is the central wavelength of the given spectral band, using the radiometric model described in [4]. Also, another parameter to be defined is the noise effective cutoff spatial frequency, $v_{\text {noise }}[$ cycle/rad], of the image spectrum SNR, which is the spatial frequency that corresponds to an image spectrum SNR value of 1. The reciprocal of $v_{\text {noise }}$ indicates a threshold noise effective spatial resolution, $\theta_{\text {th }}$ $[\mathrm{rad}]$, under which the noise exceeds the signal in a given image; i.e., if the angular spatial resolution gets smaller than $\theta_{t h}$, the noise recorded in the image due to scattering will exceed the signal level. Figure (4) shows the radiallyaveraged image spectrum $\mathrm{SNR}, S N R_{\text {rad }}$, versus the spatial frequency, $v$, for the fog aerosol models at $z=300[\mathrm{~m}]$ for the fog aerosol models according to Eqs. (15 - 18). The diffraction-limited plots, shown in figure (4), assume the absence of atmospheric aerosols; $K_{s c a}=0$. Table (3) lists the estimated values of $v_{\text {noise }}$ $\left[\right.$ cycle/rad] and the corresponding values of $\theta_{t h}[\mathrm{rad}]$, of the image spectrum SNR for the atmospheric aerosol models, at each spectral band as shown in figure (4). The values of $\theta_{t h}$ which is listed in Table (3) indicate that for each atmospheric aerosol model $\theta_{t h}$ at the visible spectral band is less than $\theta_{t h}$ is at the NIR spectral band, indicating a better spatial resolution of the imaging system at shorter wavelengths, and also a better SNR. It is also noticed that for each spectral band, $\theta_{t h}$ is minimum for the moderate radiation fog aerosol model, and $\theta_{t h}$ is maximum for the heavy advection fog aerosol model, indicating a better spatial resolution for the moderate radiation fog aerosol models, that are characterized by a relatively lower forward-scattering, compared with the heavy advection fog aerosol models. This notice is consistent with the values of the meteorological ranges, $M$, listed in Table (2), for each atmospheric aerosol 
model at the visible spectral band, where, as $M$ decreases, $\theta_{t h}$ increases indicating a reduction in the image spectrum SNR [4].

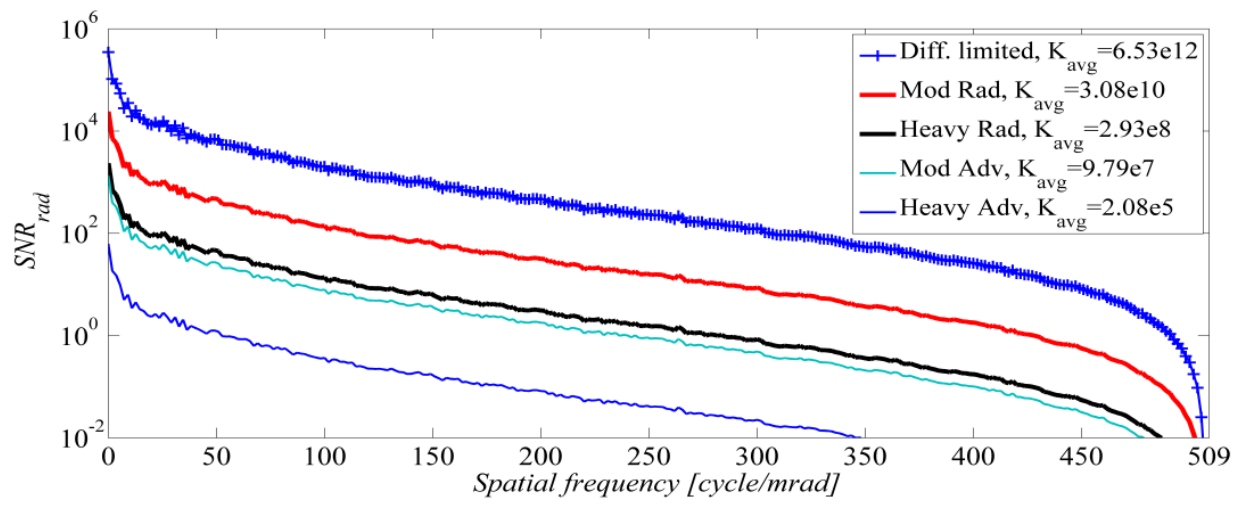

(a)

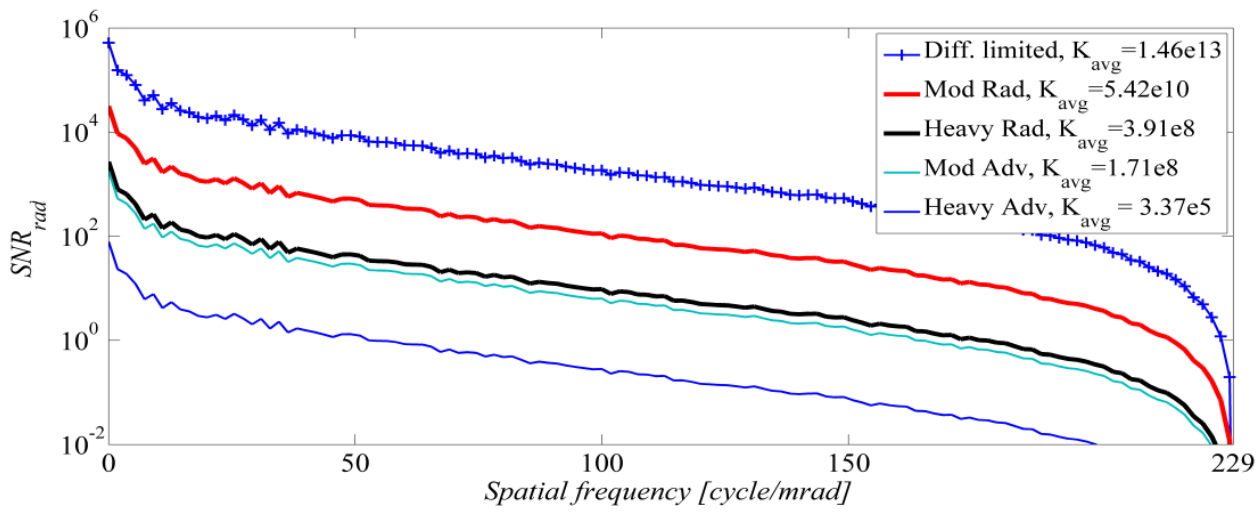

(b)

Figure (4) Radially-averaged image spectrum signal-to-noise ratio, $S N R_{\text {rad }}$, for the fog aerosol models at the visible and NIR spectral wavelength bands, for an imaging system with $D=28$ $[\mathrm{cm}]$, at $\mathrm{z}=300[\mathrm{~m}]$.

(a) Visible band $\left(\lambda_{c}=0.55 \mu \mathrm{m}\right)$, (b) NIR band $\left(\lambda_{c}=1.22 \mu \mathrm{m}\right)$,

Table (3) The estimated noise effective cutoff spatial frequency, $v_{\text {noise }}[\mathrm{cycle} / \mathrm{rad}]$, of the image-spectrum SNR for the atmospheric aerosol models, for each spectral band as shown in figure (4), and the corresponding threshold noise effective spatial resolution, $\theta_{t h}[\mathrm{rad}]$.

\begin{tabular}{lcccc}
\hline & \multicolumn{2}{c}{$\begin{array}{c}\text { Visible spectral band } \\
\lambda_{c}=0.55[\mu \mathrm{m}]\end{array}$} & \multicolumn{2}{c}{$\begin{array}{c}\text { NIR spectral band } \\
\lambda_{c}=1.22[\mu \mathrm{m}]\end{array}$} \\
\cline { 2 - 5 } Fog aerosol models & $\begin{array}{c}v_{\text {noise }} \\
{[\text { cycle/mrad }]}\end{array}$ & $\begin{array}{c}\theta_{\text {th }} \\
{[\mu \mathrm{rad}]}\end{array}$ & $\begin{array}{c}v_{\text {noise }} \\
{[\text { cycle/mrad }]}\end{array}$ & $\begin{array}{c}\theta_{\text {th }} \\
{[\mu \mathrm{rad}]}\end{array}$ \\
\hline No aerosols & 491 & 2.04 & 223.3 & 4.478 \\
Moderate radiation fog & 428 & 2.34 & 196.0 & 5.102 \\
Heavy radiation fog & 317 & 3.15 & 156.0 & 6.410 \\
Heavy radiation fog & 276 & 3.62 & 131.0 & 7.634 \\
Heavy advection Fog & 46 & 21.74 & 24.5 & 40.816 \\
\hline
\end{tabular}




\section{Conclusion}

This paper investigates the degrading effect due to light scattering which is provided by atmospheric fog aerosol models that can significantly affect the quality of image acquired by electro-optical imaging systems. We clarified the basic calculation of the atmospheric properties including the extinction coefficient, scattering coefficient, absorption coefficient, and scattering albedo, which contribute to the average point spread function due to various fog aerosol models. The PSF model confirms the concentration of the coherent PSF, resulting from the acquired direct radiation, into an attenuated Airy pattern, whereas the incoherent PSF, that is due to the acquired scattered radiation, is spread throughout the image plane regarding to the fact that scattering has the effect of broadening the angle at which the scattered light arrives at the imaging system compared to the direct light. Also, an increased atmospheric optical depth attenuates the level of the direct PSF and increases the level of the scattered PSF leading to a reduced image contrast. The level of the scattered radiation relative to the level of the direct radiation coming from a source determines the shape of the point spread function, where as long as $P S F_{d i r}>$ $P S F_{s c a}$, it could be possible to get a reasonable image quality of a distant source. However, for an increasing atmospheric optical depth $\tau$, the image contrast decreases gradually until the image is completely unseen for $P S F_{s c a} \geq P S F_{d i r}$. Finally, the analysis of fog aerosol models leads to the important conclusion of the limiting effect of aerosol scattering on the spatial resolution of the imaging system. The other metric that emphasize the degrading effect due to fog aerosols is the calculation of the signal-to-noise ratio recorded in a detected image and hence, clarifies quantitatively the effect of atmospheric scattering on image quality. We used a radiometric model for estimating the mean numbers of direct and scattered photons detected by an imaging system from an extended target, for a horizontal near-ground imaging scenario, upon propagation through various fog aerosol models in the visible, and NIR spectral bands. We incorporated the calculation of the effective PSF due to the direct and scattered radiation acquired by an imaging system to estimate the mean numbers of direct and scattered photons detected by an imaging system, which are then utilized to calculate the image spectrum SNR. Finally, we demonstrated a technique for predicting the resolution limit of an imaging system working in the presence of fog and haze atmospheric aerosol models as a function of the optical system parameters, the scattering medium, and the signal level which show a reduction in the value of the noise effective spatial frequency, and consequently an increase in the noise effective spatial resolution, for images taken through fog aerosol media that are characterized by strong forward-scattering, hence emphasize the degrading effect of fog aerosol models on the spatial resolution of imaging systems operating in the visible, and NIR spectral bands. 


\section{REFERENCES}

1. C. F. Bohren, and D. R. Huffman, Absorption and scattering of light by small particles. 2004: WILEY-VCH Verlag GmbH \& Co. KGaA, Weinheim.

2. N. S. Kopeika, and D. Sadot, Imaging through the atmosphere: practical instrumentation-based theory and verification of aerosol modulation transfer function. Journal of the Optical Society of America A, 1993. 10 (1): p. 172-179.

3. M. E. Hanafy, M. C. Roggemann, and D. O. Guney, Detailed effects of scattering and absorption by haze and aerosols in the atmosphere on the average point spread function of an imaging system. Journal of the Optical Society of America A, 2014. 31 (6): p. 1312 - 1319.

4. M. E. Hanafy, M. C. Roggemann, and D. O. Guney, Estimating the image spectrumsignal-to-noise ratio for imaging through scattering media. Optical Engineering, 2015. 54 (1): p. 013102-1 - 013102-10.

5. R. C. Shirkey, and S. G. O'Brien, An Analysis of Atmospheric Aerosol Scattering for Mid-Infrared Systems. 2006, U.S. Army Research Laboratory, White Sands Missile Range, NM 88002-5501.

6. R. C. Shirkey, and D. H. Tofsted, High Resolution Electro-Optical Aerosol Phase Function Database PFNDAT2006. 2008, U.S. Army Research Laboratory, White Sands Missile Range.

7. $\quad$ E. P. Shettle, and R. W. Fenn, Models for the Aerosols of the Lower Atmosphere and the Effects of Humidity Variations on Their Optical Properties. 1979, U.S. Air Force Geophysics Laboratory, Hanscom Air Force Base, MA.

8. H. C. Hulst, Light scattering by small particles. 1981, New York: Dover Publications, Inc.

9. K. N. Liou, An Introduction to Atmospheric Radiation. 2002: Academic Press.

10. A. Ishimaru, Wave Propagation and Scattering in Random Media. The IEEE/OUP Series, ed. D.G. Dudley. 1997, New York: IEEE Press and Oxford University Press.

11. R. G. Grainger, Some Useful Formulae for Aerosol Size Distributions and Optical Properties. 2012, University of Oxford.

12. M. T. Eismann, and D. A. LeMaster, Aerosol modulation transfer function model for passive long-range imaging over a nonuniform atmospheric path. Optical Engineering, 2013. 52 (4): p. 046201-1-046201-13.

13. B. B. Dor et al., Atmospheric scattering effect on spatial resolution of imaging systems. Journal of the Optical Society of America A, 1997. 14 (6): p. 1329-1337.

14. L. R. Bissonnette, Imaging through fog and rain. Optical Engineering, 1992. 31 (5): p. 1045-1052.

15. M. C. Roggemann, and B. M. Welsh, Imaging Through Turbelence. 1996: CRC Press. 